\title{
ANÁLISE DE RISCO DE CONTAMINAÇÃO DAS ÁGUAS SUBTERRÂNEAS E SUPERFICIAIS DA REGIÃO DE PETROLINA (PE) E JUAZEIRO (BA)
}

\author{
VERA L. FERRACINI ${ }^{*}$ \\ MARIA C. Y. P. PESSOA** \\ ADERALDO S. SILVA*** \\ CLÁUDIO A. SPADOTTO****
}

\begin{abstract}
Analisou-se o potencial de contaminação das águas subterrâneas e superficiais do Submédio do Rio São Francisco por pesticidas aplicados nas culturas de manga e uva, mediante critérios da Environmental Protection Agency, do índice de GUS e critérios propostos por GOSS. Todos os critérios utilizados levam em consideração as propriedades dos produtos aplicados, não demandando custos elevados nem muito tempo para o levantamento das informações e para a avaliação do potencial de contaminação. Os resultados obtidos reforçam a importância de disponibilizar informações sobre as propriedades físico-químicas dos pesticidas, principalmente o coeficiente de adsorção, cujo valor permite a previsão da mobilidade do composto no solo. Este fator, integrado ao conhecimento do tempo de degradação do produto até a metade de sua concentração inicial (meia-vida) no solo, fornece informações sobre a sua influência no potencial de contaminação das águas. Os resultados deste trabalho propiciam o conhecimento dos pesticidas com maior potencial de contaminação dos recursos hídricos, os quais devem ser priorizados no monitoramento ambiental "in loco".
\end{abstract}

PALAVRAS-CHAVE: ÁGUA; ÁGUAS SUBTERRÂNEAS; ÁGUAS SUPERFICIAIS; ÁGUACONTAMINAÇÃO.

* Ph.D. Química Orgânica, Embrapa Meio Ambiente, Jaguariúna, SP. (e-mail: veraf@cnpma.embrapa.br).

** Ph.D. Engenharia Elétrica, Automação e Matemática Aplicada, Embrapa Meio Ambiente, Jaguariúna - SP. (e-mail: young@cnpma.embrapa.br).

*** Ph.D. Impacto Ambiental em Agricultura Irrigada, Embrapa Meio Ambiente, Jaguariúna, SP. (e-mail: aderaldo@cnpma.embrapa.br).

**** Ph.D. Engenharia Agronômica, Embrapa Meio Ambiente, Jaguariúna, SP. (e-mail: spadotto@cnpma.embrapa.br). 


\section{INTRODUÇÃO}

A qualidade da água do semi-árido brasileiro depende, principalmente, do controle dos resíduos urbanos ribeirinhos presentes nos grandes reservatórios, barragens, açudes, rios e lagos, bem como das atividades agroindustriais e de mineração realizadas ao longo dos seus limites. Esta região apresenta agricultura irrigada intensiva de fruteiras, cujo retorno social é expressivo para a própria fixação do homem no campo.

No Submédio do Rio São Francisco as fruteiras irrigadas de manga e uva são exploradas por pequenos e grandes produtores que têm contribuído nos últimos anos com cifras expressivas para a economia nacional, mediante exportação destes produtos. Considerando que as condições climáticas locais favorecem sobremaneira o aparecimento de pragas e doenças e, dada as características físicas dos solos (na sua maioria arenosos, sendo encontrados também podzólicos, latossolos e vertissolos), a alta utilização de insumos e pesticidas é imprescindível para assegurar a produtividade agrícola. Estes, por sua vez, oferecem risco de aumento nos níveis de nitrato, fosfato e nas concentrações residuais dos pesticidas, ou de seus metabólitos, que podem comprometer a qualidade do solo e das águas superficiais e subterrâneas. Neste sentido, salienta-se também a forte demanda por água para consumo humano e irrigação na região, cuja qualidade deve ser analisada a fim de evitar desperdícios e problemas para a saúde humana e animal.

O uso incorreto de produtos químicos em áreas agrícolas representa grande ameaça ao meio ambiente. A natureza orgânica das moléculas de muitos produtos permite sua degradação, sendo necessário estudar os destinos e as conseqüências do transporte destas moléculas e seus resíduos (MATTOS e SILVA, 1999).

Segundo FERRAZ (1996) os recursos hídricos agem como integradores dos processos biogeoquímicos de qualquer região. Sendo assim, quando os pesticidas são introduzidos, os recursos hídricos, sejam superficiais ou subterrâneos, aparecem como o principal destino final dos pesticidas. Na maioria das vezes, a concentração dos pesticidas em água é baixa, em parte por serem, geralmente, pouco solúveis e em parte devido ao efeito de diluição. Entretanto, mesmo nestas concentrações, tais produtos representam riscos para algumas espécies de organismos aquáticos, que podem concentrá-los em até 1000 vezes.

A interação de solos e pesticidas freqüentemente tem dificultado a avaliação do comportamento de determinado pesticida no ambiente. Estes compostos apresentam propriedades como meia-vida $\left(\mathrm{DT}_{50}\right)$, coeficiente de adsorção à matéria orgânica (Koc) e solubilidade, que 
interagem com as propriedades dos solos tais como teor da matéria orgânica, potencial de erosão e propriedades hidráulicas (GOSS, 1992). Segundo GOSS (1992) em solos orgânicos raramente ocorre perda de pesticida por "escoamento superficial" e lixiviação, e pesticidas com Koc acima de $300 \mathrm{~mL} . \mathrm{g}^{-1}$ são fortemente adsorvidos pela matéria orgânica. $\mathrm{O}$ potencial de perda de pesticidas pela água superficial ou lixiviação depende da combinação de pesticida, solo, clima e fatores de manejo. Assim, a análise de pesticidas utilizados na cadeia produtiva destas culturas possibilita a identificação dos produtos que podem oferecer risco potencial ou apresentar potencial de contaminação das águas superficiais e subterrâneas. Várias são as formas de elaborar tal análise, muitas das quais demandam informações detalhadas, como as obtidas por simulações da dinâmica de pesticidas no perfil vertical dos solos. Outro modo de se analisar o potencial de contaminação de águas subterrâneas envolve as próprias características dos produtos, usando os critérios de "screening" da Environmental Protection Agency (EPA) (COHEN et al., 1995) ou o índice de GUS (Groundwater Ubiquity Score) (FUNARI et al., 1991). O potencial de contaminação das águas superficiais também pode ser previsto pelas características dos pesticidas utilizando-se os critérios propostos por GOSS (1992) e GUSTAFSON (1989). Estes critérios classificam o risco de contaminação em alto, médio e baixo em função do transporte de pesticidas em sedimentos e dissolvidos em água.

O presente trabalho evidencia o potencial de contaminação de águas superficiais e subterrâneas por pesticidas utilizados nas fruteiras irrigadas de manga e uva do Submédio do São Francisco.

\section{MATERIAL E MÉTODOS}

\section{1 ÁREA DE ESTUDO}

A área estudada constitui-se em região irrigada do Submédio do São Francisco, localizada em latitude $9,24^{\circ} \mathrm{S}$ e longitude $40,30^{\circ} \mathrm{N}$, com elevação de $375 \mathrm{~m}$, na região de Petrolina/PE e Juazeiro/BA. Apesar da baixa umidade relativa (média anual inferior a $60 \%$ ) e pluviosidade média (450 mm), a região prospera na produção de frutas graças às técnicas de irrigação e de aplicações de agrotóxicos para o controle de pragas e doenças das culturas.

As fruteiras de manga e uva predominam na região, assim como os solos do tipo areias quartzosas e outros com limitações para o uso agrícola em função da escassez de água, tais como os podzólicos, latossolos vermelho-escuro e latossolos vermelho-amarelo, e aqueles com 
probabilidades de fendilhamentos e ressecamentos, como os vertissolos.

As areias quartzosas apresentam textura arenosa ou franco arenosa nos horizontes até, no mínimo, a profundidade de $150 \mathrm{~cm}$ a partir da superfície do solo ou até contato lítico. São praticamente isentos de minerais primários alteráveis e, conseqüentemente, menos resistentes a intemperismo (EMBRAPA-CNPS, 1999). Os vertissolos, em áreas irrigadas ou mal drenadas (sem fendas aparentes) apresentam expansibilidade linear de $6 \mathrm{~cm}$ ou mais. Também apresentam fendas verticais no período seco, com pelo menos $1 \mathrm{~cm}$ de largura, atingindo, no mínimo $50 \mathrm{~cm}$ de profundidade, exceto no caso de solos rasos, cujo limite mínimo é de $30 \mathrm{~cm}$ de profundidade (EMBRAPA-CNPS, 1999). Estes solos, em particular, favorecem a ocorrência de processos de lixiviação somente pelas suas características físicas. Além disso, os lençóis subterrâneos superficiais encontram-se localizados a partir de $1,5 \mathrm{~m}$ de profundidade, portanto, a aplicação de agrotóxicos nas culturas deve ser monitorada com o intuito de prevenir a contaminação de águas subterrâneas e superficiais.

Os latossolos são, geralmente, solos em avançado estágio de intemperização e, desta forma, visivelmente destituídos de minerais primários e secundários. São geralmente muito profundos e fortemente ácidos, com baixa saturação por bases, distróficos ou álicos, características estas que na região semi-árida diferenciam-se por apresentar caráter de solos com média e até mesmo alta saturação por base (EMBRAPA-CNPS, 1999). A diversidade reduzida em função das extensas áreas de manga e uva e a constante exploração do solo e plantas eleva a necessidade do uso de agrotóxicos.

Entre os sistemas de irrigação utilizados na região citam-se como predominantes na cultura de manga os do tipo sulco, microaspersão e aspersão convencional. Já na cultura de uva somente os do tipo aspersão convencional e microaspersão são usados (EMBRAPA MEIO AMBIENTE, 1999a). O manejo da água é fundamental para o desenvolvimento das culturas, cuja necessidade de água depende do seu respectivo desenvolvimento fenológico e do sistema de irrigação selecionado. Sob condições de irrigação por sulco e por aspersão convencional, o nível de água disponível no solo deve ser mantido acima de 50\%, enquanto que sob irrigação localizada entre 80 a 100\% (SOARES, 1995).

\subsection{LEVANTAMENTO DE PESTICIDAS UTILIZADOS NAS CULTURAS DE MANGA E UVA}

A seleção dos pesticidas para este estudo foi realizada com aplicação de questionários junto aos produtores das culturas de manga e 
uva da região (EMBRAPA MEIO AMBIENTE, 1999a, b). No levantamento realizado verificou-se a predominância de compostos da classe dos organofosforados, $36 \%$, e dos benzimidazóis, $24 \%$. Os carbamatos e os piretróides são aplicados com freqüência em torno de $12 \%$. Já os compostos clorados são pouco utilizados na região com freqüência em torno de $6 \%$.

$\mathrm{Na}$ Tabela 1 constam as propriedades dos pesticidas significativas para este trabalho como: meia-vida no solo, solubilidade em água e coeficiente de adsorção obtidos na literatura (WORTHING \& HANCE, 1991 e WAUCHOPE et al., 1992).

\section{TABELA 1 - CARACTERÍSTICAS DOS PESTICIDAS UTILIZADOS NAS FRUTEIRAS DE MANGA E UVA}

\begin{tabular}{|c|c|c|c|c|c|}
\hline Compostos & $\begin{array}{c}\text { Solubilidade em } \\
\text { gua }\left(\mathrm{g} \cdot \mathrm{m}^{-3}\right)\end{array}$ & $\operatorname{Koc}\left(m L g^{-1}\right)$ & $\begin{array}{l}\mathrm{DT}_{50} \text { solo } \\
\text { (dias) }\end{array}$ & $\begin{array}{c}\mathrm{K}_{\mathrm{H}} \\
(\text { Pa.m 3.mol } \\
\text { (-1) }\end{array}$ & GUS \\
\hline Acefato & 818.000 & 3,00 & 2,00 & $0,50 \times 10^{-4}$ & 1,06 \\
\hline Abam ectim & 5,00 & 5.000 & 28,00 & 0,10 & 0,44 \\
\hline Benomil & 2,00 & 1.900 & 67,00 & $1,90 \times 10^{-6}$ & 1,32 \\
\hline Benzilato & - & - & - & - & - \\
\hline Carbendazin & 28,00 & 400 & $30-150$ & $1,60 \times 10^{-2}$ & 2,91 \\
\hline Cimoxamil & $890(\mathrm{pH} \mathrm{5)}$ & - & $<14$ & $1,60 \times 10^{-5}$ & - \\
\hline Ciproconazole & 11.000 & - & - & 3,40 & - \\
\hline Clorotalonil & 0,60 & 1.380 & 30,00 & $0,06 \times 10^{3}$ & 1,27 \\
\hline Clorpirifos & 0,40 & 6.070 & 30,00 & $1,90 \times 10^{-6}$ & 0,32 \\
\hline Cianamide & - & - & - & - & - \\
\hline Difenoconazole & 3,30 & - & - & 14,70 & - \\
\hline Deltam etrina & $<0,20 \times 10^{-4}$ & 460.000 & $<23$ & $>0,50$ & $-2,26$ \\
\hline Dimetoato & 39.800 & 20,00 & 7,00 & $0,10 \times 10^{-3}$ & 2,28 \\
\hline Diuron & 42,00 & 480,00 & 90,0 & $5,10 \times 10^{-5}$ & 2,57 \\
\hline Fenarimol & 14,00 & 600,00 & 360,0 & $6,90 \times 10^{-4}$ & 3,13 \\
\hline Fenitrotion & 21,00 & 2.000 & 0,20 & $2,10 \times 10^{-3}$ & $-0,50$ \\
\hline Fention & 4,20 & 1.500 & 34,00 & $0,60 \times 10^{-6}$ & 1,26 \\
\hline Fosetil & 120.000 & 20,00 & 0,30 & $1,20 \times 10^{-5}$ & 1,60 \\
\hline Glifosato & $900.000 \mathrm{E}$ & $24.000 \mathrm{E}$ & 47,00 & 0,00 & $-0,64$ \\
\hline Iprodione & 13,90 & 700,00 & 14,00 & $3,10 \times 10^{-4}$ & 1,33 \\
\hline Im idaclopride & - & - & - & - & - \\
\hline Mancozeb & 6,00 & $>2.000$ & 70,00 & 0,00 & 1,29 \\
\hline Maneb & $6 \mathrm{E}$ & $>2.000$ & 70,00 & 0,00 & 1,29 \\
\hline
\end{tabular}

continua... 
continuação

\begin{tabular}{|c|c|c|c|c|c|}
\hline Compostos & \begin{tabular}{|c|} 
Solubilidade em \\
gua $\left(\mathrm{g}^{-3} \mathrm{~m}^{-3}\right)$
\end{tabular} & $\operatorname{Koc}\left(\mathrm{mL} \mathrm{g}^{-1}\right)$ & $\begin{array}{l}\mathrm{DT}_{50} \text { solo } \\
\text { (dias) }\end{array}$ & $\underset{(\text { Pa.m3.mol }}{\mathrm{K}_{\mathrm{H}}}$ & GUS \\
\hline Metalaxil & 8.400 & $50 \mathrm{E}$ & 70,00 & $2,50 \times 10^{-4}$ & 4,23 \\
\hline Metamidof $\mathrm{s}$ & 1.000.000E & 5,00 & 6,00 & $1,40 \times 10^{-5}$ & 2,57 \\
\hline Metidation & 220,00 & $400 \mathrm{E}$ & 7,00 & $0,20 \times 10^{-4}$ & 1,19 \\
\hline Metil paration & 60,00 & 5.100 & 5,00 & $8,70 \times 10^{-3}$ & 0,20 \\
\hline Metomil & 58.000 & 72,00 & 30,00 & $0,09 \times 10^{-3}$ & 3,16 \\
\hline Miclobutanil & 142,00 & 500,00 & 66,00 & 0,40 & 2,37 \\
\hline Monocrotof s & 1.000 .000 & $1 \mathrm{E}$ & 30,00 & $2,07 \times 10^{-7}$ & 5,91 \\
\hline Tebuconazole & $50(\mathrm{pH} 7)$ & 2.500 & 403 & $2,00 \times 10^{-5}$ & 1,56 \\
\hline Tiofanato metil & 3,50 & 1.830 & 106 & $1,30 \times 10^{-1}$ & 1,49 \\
\hline Triclorfon & 120.000 & 10,00 & 10,00 & $0,03 \times 10^{-3}$ & 3,00 \\
\hline Paclobutrazol & 35,00 & 66,00 & 180 & $3,90 \times 10^{-\prime}$ & 4,92 \\
\hline Paraquat & 620.000 & $1.000 .000 \mathrm{E}$ & $1.000 \mathrm{E}$ & 0,00 & $-6,00$ \\
\hline Paration Etil & 24,00 & $5000 \mathrm{E}$ & 14,0 & $8,00 \times 10^{-3}$ & 0,34 \\
\hline Pirazof $s$ & - & - & - & - & - \\
\hline Plocloraz & 34,00 & $500 \mathrm{E}$ & 120,00 & $1,60 \times 10^{-3}$ & 2,71 \\
\hline Thiran & 30,00 & 670,00 & 15,00 & $9,30 \times 10^{-3}$ & 1,38 \\
\hline
\end{tabular}

$\mathrm{E}=$ Valor estimado.

- = Valor não encontrado na literatura.

DT50 = Meia-vida do produto.

$\mathrm{KH}=$ Constante de Henry.

GUS = Groundwater Ubiquity Score.

Koc $=$ Coeficiente de adsorção.

A maioria dos valores da constante de Henry e do índice de GUS (WORTHING \& HANCE, 1991; WAUCHOPE et al., 1992) foram calculados por não terem sido encontrados na literatura. Para calcular estes valores foram empregados os dados da Tabela 1 , sendo utilizados para os dados apresentados (na Tabela) com sinais $>$ ou $<0$ seu valor numérico para efeito dos cálculos.

\subsection{ANÁLISE DE RISCO DE CONTAMINAÇÃO DAS ÁGUAS SUBTERRÂNEAS}

Os critérios de "screening" da EPA (COHEN et al., 1995) e o índice de GUS (FUNARI et al., 1991) foram utilizados para avaliar o risco 
de contaminação de águas subterrâneas. Os critérios da EPA envolvem:

a) solubilidade em água $>30 \mathrm{mg} \cdot \mathrm{mL}^{-1}$;

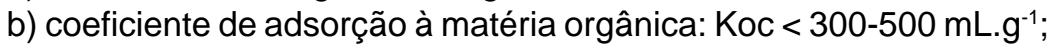

c) constante de Henry: $\mathrm{KH}<10^{-2} \mathrm{~Pa} \cdot \mathrm{m}^{3} \cdot \mathrm{mol}^{-1}$;

d) meia-vida no solo ( DT50 no solo): > 14-21 dias;

e) meia-vida na água ( DT50 na água) > 175 dias.

Neste critério também devem ser consideradas as condições de campo que favorecem a percolação no solo, ou seja, pluviosidade anual $>250 \mathrm{~mm}$; presença de solo poroso e presença de aqüífero não-confinado.

A análise conforme o índice de GUS é realizada mediante fórmula matemática tendo como parâmetros os valores de meia-vida do composto no solo e do coeficiente de adsorção à matéria orgânica do solo, de acordo com a seguinte equação:

na qual:

$$
\text { GUS }=\log \left(D_{50} \text { solo }\right) \times(4-\log (\text { Koc }))
$$

DT50 = Meia-vida do produto no solo.

Koc = Coeficiente de adsorção à matéria orgânica no solo.

Uma vez determinado o índice de GUS para cada pesticida, os mesmos são classificados em função dos seguintes critérios:
a) GUS $=1,8$
Não sofre lixiviação.
b) $1,8<$ GUS $<2,8$
Faixa de transição.
c) GUS $\geq 2,8$
Provável lixiviação.

O valor de GUS serve como ferramenta auxiliar para identificação de pesticidas a serem priorizados nas atividades de monitoramento ambiental in loco. Permite a identificação do provável compartimento em que o composto deve ser monitorado no caso de sedimento ou água.

\subsection{ANÁLISE DE RISCO DE CONTAMINAÇÃO DAS ÁGUAS SUPERFICIAIS}

Para avaliar o risco de contaminação de águas superficiais é utilizado o método de Goss (GOSS, 1992) que classifica o potencial de contaminação em alto, médio e baixo em função do transporte do pesticida adsorvido a colóides de solo ou dissolvido em água (Quadros 1 e 2). Os compostos não-enquadrados em nenhum dos critérios são considerados como tendo potencial médio para contaminarem águas superficiais. 


\section{QUADRO 1 - CRITÉRIOS PARA DETERMINAÇÃO DO POTENCIAL DE TRANSPORTE ASSOCIADO AO SEDIMENTO}

\begin{tabular}{|c|c|}
\hline ALTO POTENCIAL & BAIXO POTENCIAL \\
\hline $\begin{array}{l}\mathrm{DT}_{50} \text { no solo } \geq 40 \text { dias } \\
\mathrm{Koc} \geq 1000 \mathrm{~mL} \cdot \mathrm{g}^{-1} \\
\mathrm{DT}_{50} \text { no solo } \geq 40 \text { dias } \\
\text { Koc } \geq 500 \mathrm{~mL} \cdot \mathrm{g}^{-1} \\
\text { Solubilidade em Agua } \leq 0,5 \mu \mathrm{g} \cdot \mathrm{mL}^{-1}\end{array}$ & 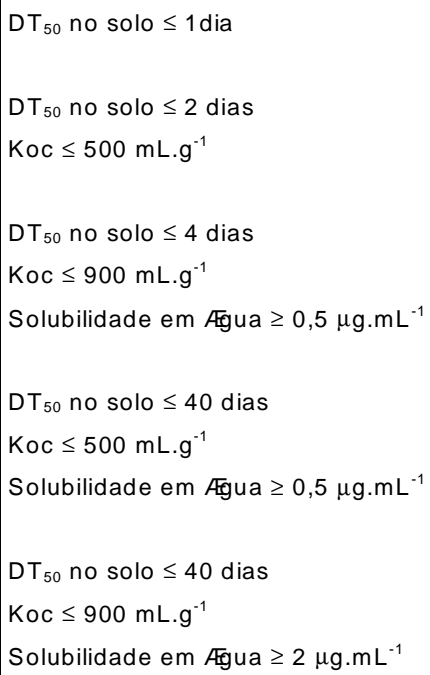 \\
\hline
\end{tabular}

\section{QUADRO 2 - CRITÉRIOS PARA DETERMINAÇÃO DO POTENCIAL DE TRANSPORTE DISSOLVIDO EM ÁGUA}

\begin{tabular}{|l|l|}
\hline ALTO POTENCIAL & BAIXO POTENCIAL \\
\hline $\begin{array}{l}\mathrm{DT}_{50} \text { no solo }>35 \text { dias } \\
\text { Koc }<100.000 \mathrm{~mL} \cdot \mathrm{g}^{-1} \\
\text { Solubilidade } \geq 1 \mu \mathrm{g} \cdot \mathrm{mL}^{-1}\end{array}$ & Koc $\geq 100.000 \mathrm{mLg}^{-1}$ \\
Koc $\leq 700 \mathrm{~mL} \cdot \mathrm{g}^{-1}$ & $\begin{array}{l}\mathrm{DT}_{50} \text { no solo } \leq 1 \text { dia } \\
\text { Koc } \geq 1000 \mathrm{~mL} \cdot \mathrm{g}^{-1}\end{array}$ \\
$10 \leq$ Solubilidade $\leq 100 \mu \mathrm{g} \cdot \mathrm{mL}^{-1}$ & $\mathrm{DT}_{50}$ no solo $<35$ dias \\
& Solubilidade $<0,5 \mu \mathrm{g} \cdot \mathrm{mL}^{-1}$ \\
\hline
\end{tabular}




\section{RESULTADOS E DISCUSSÃO}

\subsection{RISCO DE CONTAMINAÇÃO DE ÁGUAS SUBTERRÂNEAS}

A classificação dos pesticidas de acordo com o potencial de contaminação de águas subterrâneas e superficiais depende da interpretação dos dados de propriedades físico-químicas dos pesticidas (Tabela 1). Apesar da lacuna existente na literatura consultada (WORTHING e HANCE, 1991 e WAUCHOPE et al., 1992) relativa às características dos compostos analisados foi possível classificar alguns pesticidas com maior probabilidade de atingir as águas subterrâneas, conforme critérios da EPA (Tabela 2). Alguns dos pesticidas analisados apresentaram elevada solubilidade em água, baixa adsorção à matéria orgânica do solo e meiavida relativamente alta. Os critérios da EPA apontam que o coeficiente de adsorção, a meia-vida no solo, a solubilidade em água e a constante de Henry $(\mathrm{KH})$ são as propriedades físico-químicas dos pesticidas mais relevantes no resultado final para sua classificação em relação à contaminação das águas.

Os dados de meia-vida em água são pouco citados na literatura, pois dependem das características do ambiente, não sendo considerados na análise.

Os princípios ativos Diuron, Metalaxil, Metomil, Monocrotofós e Plocloraz atenderam a todos os critérios de avaliação estabelecidos, indicando potencial de contaminação de águas subterrâneas.

A meia-vida do Acefato, Dimetoato, Fosetil, Metamidofós, Metidation e Triclorfon no solo não atenderam aos critérios da avaliação, mas as demais propriedades sim, o que os coloca sob suspeita de se tornarem agentes causadores de contaminação de águas subterrâneas. Esta contaminação poderá ocorrer quando as condições do solo não favorecerem a degradação e/ou adsorção. O Tebuconazole, cujo coeficiente de adsorção não atende aos critérios da avaliação, está também sob suspeita de se tornar agente causador de contaminação de águas subterrâneas.

Os resultados das análises do potencial de contaminação das águas subterrâneas, baseadas nos critérios da EPA, evidenciaram vinte e dois compostos com potencial de contaminação (Tabela 2).

Comparando-se os resultados da análise do potencial de contaminação de águas subterrâneas, baseados nos critérios da EPA (Tabela 2), com o índice de GUS é possível perceber que o coeficiente de adsorção e a meia-vida no solo são as propriedades mais relevantes dos pesticidas. Neste estudo comparativo, o número de compostos com potencial de contaminação das águas subterrâneas ficou reduzido para dez (Tabela 3). 


\section{TABELA 2 - AVALIAÇÃO DE RISCO DE CONTAMINAÇÃO DE ÁGUAS SUBTERRÂNEAS CONFORME CRITÉRIOS DA EPA}

\begin{tabular}{|c|c|c|c|c|c|}
\hline Compostos & $\begin{array}{l}\text { Solubilidade } \\
\text { em gua } \\
\left(\mathrm{g} \cdot \mathrm{m}^{-3}\right)\end{array}$ & $\begin{array}{c}\text { Koc } \\
(\mathrm{mL} \cdot \mathrm{g}-1)\end{array}$ & $\begin{array}{l}\text { DT }_{50} \text { Solo } \\
\text { (dias) }\end{array}$ & $\begin{array}{c}\mathrm{K}_{\mathrm{H}} \\
\text { (Pa.m3.mol-1) }\end{array}$ & $\begin{array}{c}\text { Critørios } \\
\text { EPA }\end{array}$ \\
\hline Acefato & 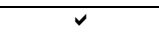 & $\checkmark$ & $\mathrm{X}$ & $\checkmark$ & PC \\
\hline Abamectim & $\mathrm{x}$ & $\mathrm{x}$ & $\checkmark$ & $\mathrm{x}$ & NC \\
\hline Benomil & $\mathrm{x}$ & $\mathrm{x}$ & $\checkmark$ & $\boldsymbol{\nu}$ & PC \\
\hline Benzilato & - & - & - & - & I \\
\hline Carbendazin & $\mathrm{x}$ & - & $\checkmark$ & $\mathrm{x}$ & I \\
\hline Cimoxamil & $\boldsymbol{v}$ & - & $\mathrm{x}$ & $\boldsymbol{\nu}$ & PC \\
\hline Ciproconazol & $\checkmark$ & - & - & $\boldsymbol{v}$ & I \\
\hline Clorotalonil & $\mathrm{x}$ & $\mathrm{x}$ & $\boldsymbol{v}$ & 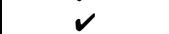 & PC \\
\hline Clorpirifos & $\mathrm{x}$ & $\mathrm{x}$ & $\checkmark$ & $\mathrm{x}$ & NC \\
\hline Cyanamide & - & - & - & - & I \\
\hline Defenoconazole & $\mathrm{x}$ & - & - & $\mathrm{x}$ & I \\
\hline Deltametrina & $\mathrm{x}$ & $x$ & $\mathrm{x}$ & $x$ & NC \\
\hline Dimetoato & $\checkmark$ & $\checkmark$ & $\mathrm{x}$ & $\checkmark$ & PC \\
\hline Diuron & $\checkmark$ & $\checkmark$ & $\checkmark$ & $\checkmark$ & PC \\
\hline Fenarimol & $\mathrm{x}$ & $\mathrm{x}$ & $\checkmark$ & $\checkmark$ & PC \\
\hline Fenotrotion & $\mathrm{x}$ & - & $\mathrm{x}$ & $\checkmark$ & I \\
\hline Fention & $x$ & $x$ & $\boldsymbol{\nu}$ & $\boldsymbol{v}$ & PC \\
\hline Fosetil & $\checkmark$ & $\checkmark$ & $\mathrm{x}$ & $\checkmark$ & PC \\
\hline Glifosato & $\checkmark$ & $x$ & $\checkmark$ & $\mathrm{x}$ & PC \\
\hline Iprodione & $x$ & $\mathrm{x}$ & $\mathrm{x}$ & $\checkmark$ & NC \\
\hline Imidaclopride & - & - & - & - & 1 \\
\hline Mancozeb & $\mathrm{x}$ & $\mathrm{x}$ & $\boldsymbol{\nu}$ & $x$ & NC \\
\hline Maneb & $\mathrm{x}$ & $\mathrm{x}$ & $\checkmark$ & $\mathrm{x}$ & NC \\
\hline Metalaxil & $\checkmark$ & $\checkmark$ & $\checkmark$ & $\checkmark$ & PC \\
\hline Metamidof s & $\checkmark$ & $v$ & $\mathrm{x}$ & $\checkmark$ & PC \\
\hline Metidation & $\checkmark$ & $\nu$ & $\mathrm{x}$ & $\checkmark$ & PC \\
\hline Metil paration & $\boldsymbol{v}$ & $x$ & $x$ & $v$ & PC \\
\hline Metomil & $\checkmark$ & $\checkmark$ & $\checkmark$ & $\checkmark$ & PC \\
\hline Miclorobutanil & $\checkmark$ & - & $\checkmark$ & $\mathrm{x}$ & I \\
\hline Monocrotof $\mathrm{s}$ & $\checkmark$ & $\checkmark$ & $\checkmark$ & $\checkmark$ & PC \\
\hline Tebuconazole & $\checkmark$ & $\mathrm{x}$ & $\checkmark$ & $\checkmark$ & PC \\
\hline Tiofanato Metil & $x$ & $x$ & $\checkmark$ & $\checkmark$ & PC \\
\hline Triclorfon & $\checkmark$ & $\checkmark$ & $x$ & $\checkmark$ & PC \\
\hline Paclobutrazol & $\checkmark$ & $\nu$ & $\checkmark$ & $\checkmark$ & PC \\
\hline Paraquat & $\checkmark$ & $\mathrm{x}$ & $\checkmark$ & $\mathrm{x}$ & PC \\
\hline Paration & $\mathrm{x}$ & $\mathrm{x}$ & $\mathrm{x}$ & $\boldsymbol{v}$ & NC \\
\hline Pirazof $\mathrm{s}$ & - & - & - & - & I \\
\hline Plocloraz & $\checkmark$ & $\checkmark$ & $\checkmark$ & $\checkmark$ & PC \\
\hline Thiran & $x$ & $x$ & $x$ & $\checkmark$ & NC \\
\hline
\end{tabular}

$\mathrm{X}=$ não atende ao critério; 4 = composto atende ao critério com potencial perigoso; $\mathrm{PC}=$ contaminante em potencial $; \mathrm{NC}=$ não-contaminante; $\mathrm{I}$ = inconclusivo; - = dado nãodisponível na literatura consultada. 


\section{TABELA 3 - AVALIAÇÃO DE RISCO DE CONTAMINAÇÃO DE ÁGUAS SUBTERRÂNEAS COMPARANDO OS CRITÉRIOS DA EPA E O ÍNDICE DE GUS}

\begin{tabular}{|c|c|c|c|}
\hline Princ pio Ativo & GUS & EPA & $\begin{array}{c}\text { Categoria } \\
\text { que atende a ambos }\end{array}$ \\
\hline Acefato & $\mathrm{NC}$ & $P C$ & 1 \\
\hline Abamectim & NC & NC & NC \\
\hline Benomil & NC & PC & I \\
\hline Benzilato & - & I & I \\
\hline Carbendazin & $\mathrm{PC}$ & 1 & I \\
\hline Cimoxamil & - & $\mathrm{PC}$ & I \\
\hline Ciproconazol & - & I & I \\
\hline Clorotalonil & NC & PC & I \\
\hline Clorpirifos & NC & NC & NC \\
\hline Cianamide & - & I & I \\
\hline Defenoconazole & - & 1 & I \\
\hline Deltametrina & NC & NC & NC \\
\hline Dimetoato & $\mathrm{T}$ & PC & PC \\
\hline Diuron & $\mathrm{T}$ & $\mathrm{PC}$ & PC \\
\hline Fenarimol & PC & PC & PC \\
\hline Fenotrotion & NC & 1 & I \\
\hline Fention & $\mathrm{NC}$ & $\mathrm{PC}$ & I \\
\hline Fosetil & NC & $\mathrm{PC}$ & I \\
\hline Glifosato & NC & PC & I \\
\hline Iprodione & NC & NC & NC \\
\hline Imidaclopride & - & I & I \\
\hline Mancozeb & NC & NC & NC \\
\hline Maneb & $\mathrm{NC}$ & $\mathrm{NC}$ & NC \\
\hline Metalaxil & PC & $\mathrm{PC}$ & PC \\
\hline Metamidof $\mathrm{s}$ & $T$ & $\mathrm{PC}$ & PC \\
\hline Metidation & NC & $\mathrm{PC}$ & 1 \\
\hline Metil paration & NC & PC & I \\
\hline Metomil & PC & $\mathrm{PC}$ & PC \\
\hline Miclorobutanil & $\mathrm{T}$ & 1 & I \\
\hline Monocrotof $\mathrm{s}$ & PC & $\mathrm{PC}$ & PC \\
\hline Tebuconazole & NC & PC & I \\
\hline Tiofanato Metil & NC & $\mathrm{PC}$ & I \\
\hline Triclorfon & $\mathrm{PC}$ & $\mathrm{PC}$ & PC \\
\hline Paclobutrazol & $\mathrm{PC}$ & $\mathrm{PC}$ & PC \\
\hline Paraquat & $\mathrm{NC}$ & $\mathrm{PC}$ & 1 \\
\hline Paration & NC & NC & NC \\
\hline Pirazof $\mathrm{s}$ & - & I & I \\
\hline Plocloraz & $T$ & PC & PC \\
\hline Thiran & NC & NC & NC \\
\hline
\end{tabular}

$\mathrm{PC}=$ contaminante em potencial; $\mathrm{NC}$ = não-contaminante; I = inconclusivo;

$\mathrm{T}$ = faixa de transição (GUS); - = dado não-disponível na literatura consultada. 
Segundo COHEN et al. (1995) os compostos classificados na faixa de transição e de lixiviação provável, de acordo com o índice de GUS, requerem investigação adicional mediante métodos mais detalhados. Segundo estes autores os princípios ativos classificados como improváveis de sofrerem lixiviação podem, seguramente, ser considerados como nãocontaminantes de águas subterrâneas. Acatando-se a afirmação de COHEN et al. (1995) e os critérios da EPA (Tabela 3) pode-se dizer que os compostos Dimetoato, Diuron, Fenarimol, Metalaxil, Metamidofós, Metomil, Monocrotofós, Paclobutrazol, Triclorfon e Plocloraz apresentam propriedades de contaminantes potenciais de águas subterrâneas na região.

Estudos para a determinação dos valores de Koc de alguns compostos em solos predominantes da região estão sendo realizados pela Embrapa Meio Ambiente com o intuito de disponibilizar informação para Paclobutrazol e Metalaxil em Latossolo Amarelo Distrófico da região. Embora raro, também podem ser encontradas algumas informações na literatura científica para Diuron em solos da região sob outros cultivos (cana-de-açúcar).

Após a avaliação de risco, os compostos Abamectim, Clorpirifós, Deltametrina, Iprodione, Mancozeb, Maneb, Paration etil e Thiran foram considerados como não-contaminantes de águas subterrâneas. Com relação aos demais, por falta de dados de suas propriedades físicoquímicas, na literatura consultada, os resultados são inconclusivos.

\subsection{RISCO DE CONTAMINAÇÃO DE ÁGUAS SUPERFICIAIS}

De acordo com os índices de Goss, usados para avaliar o potencial de determinado pesticida em atingir águas superficiais, os compostos são classificados em dois grupos: os que podem ser transportados dissolvido em água e os que são transportados associados ao sedimento em suspensão.

As informações disponibilizadas na Tabela 4 refletem que grande número de pesticidas apresenta potencial de ser transportado dissolvido em água como: Benomil, Dimetoato, Diuron, Fenarimol, Fention, Glifosato, Iprodione, Metalaxil, Paclobutrazol, Plocloraz, Tebuconazole e Thiran. Entre estes, ressalta-se que Benomil, Fention, Glifosato, Iprodione, Tebuconazole e Thiran não são contaminantes potenciais de águas subterrâneas. Por apresentarem alta afinidade pela matéria orgânica, Benomil, Glifosato e Tebuconazole podem ser transportados superficialmente, tanto dissolvidos em água como associados ao sedimento. 
Verifica-se pela Tabela 1 que quanto menor o valor de meia-vida menos lixiviável o composto se apresenta. Apesar de apresentar valor de DT50 de 34 dias, o Fention revela potencial de lixiviação para águas superficiais pelo valor de Koc de $1.500 \mathrm{~mL} . \mathrm{g}^{-1}$. Dentre os produtos com alto potencial de transporte associado ao sedimento destacam-se 0 Paraquat e o Tebuconazole, devido à elevada meia-vida no solo (aproximadamente 1000 dias e 430 dias, respectivamente). Os valores de

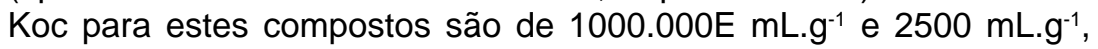
respectivamente.

\section{TABELA 4 - CLASSIFICAÇÃO DOS COMPOSTOS DE ACORDO COM SEU POTENCIAL PARA CONTAMINAÇÃO DE ÁGUAS SUPERFICIAIS}

\begin{tabular}{|c|c|}
\hline \multicolumn{2}{|c|}{ ALTO POTENCIAL DE CONTAMINA`^O DE`GUA SUPERFICIAL } \\
\hline $\begin{array}{l}\text { Dissolvidos em Agua } \\
\text { Benomil } \\
\text { Dimetoato } \\
\text { Diuron } \\
\text { Fenarimol } \\
\text { Fention } \\
\text { Glifosato } \\
\text { Iprodione } \\
\text { Metalaxil } \\
\text { Tebuconazole } \\
\text { Paclobutrazol } \\
\text { Plocloraz } \\
\text { Thiran }\end{array}$ & $\begin{array}{l}\text { Associados ao sedimento em suspens } \text { o }^{\text {o }} \\
\text { Benomil } \\
\text { Glifosato } \\
\text { Mancozeb } \\
\text { Maneb } \\
\text { Paraquat } \\
\text { Tebuconazole } \\
\text { Tiofanato metil }\end{array}$ \\
\hline \multicolumn{2}{|c|}{ BAIXO POTENCIAL DE CONTAMINA``O DE` GUA SUPERFICIAL } \\
\hline $\begin{array}{l}\text { Dissolvidos em Agua } \\
\text { Clorotalonil } \\
\text { Clorpirif s } \\
\text { Deltametrina } \\
\text { Paration etil } \\
\text { Paraquat }\end{array}$ & $\begin{array}{l}\text { Associados ao sedimento em suspens } 0 \\
\text { Acefato } \\
\text { Cimoxanil } \\
\text { Dimetoato } \\
\text { Fenitrotion } \\
\text { Fosetil } \\
\text { Iprodione } \\
\text { Metamidof s } \\
\text { Metidation } \\
\text { Metomil } \\
\text { Monocrotof s } \\
\text { Plocloraz } \\
\text { Thiran } \\
\text { Triclorfon }\end{array}$ \\
\hline
\end{tabular}


Considerando-se as características do clima e do solo da região do Submédio do São Francisco e por se tratar de área irrigada, os riscos de contaminação das águas superficiais e subterrâneas não podem ser desprezados. Somam-se a este fator de risco as características físicas dos solos da região (predominantemente areias quartzosas, podzólicos, latossolos vermelho-escuro, latossolos vermelho-amarelo e vertissolos) que propiciam a lixiviação dos produtos para camadas mais profundas, favorecendo contaminações subterrâneas e superficiais.

Observou-se também que o teor de carbono orgânico foi o parâmetro que exerceu maior influência no fluxo descendente de pesticidas nos solos, pois quanto maior a presença de matéria orgânica no solo, menor o seu potencial de lixiviação e, conseqüentemente, da contaminação de água subterrânea. Para solos com teores similares de matéria orgânica, as maiores vulnerabilidades para a contaminação das águas acontecem nos solos em que o lençol freático é menos profundo.

No caso de pesticidas com valores reduzidos de Koc, a meiavida passa a exercer influência na lixiviação. Quanto maior o valor de DT50 maior será o potencial de contaminação das águas, dado o maior tempo necessário para a degradação do pesticida no solo e sua conseqüente permanência no ambiente.

Não deve ser desprezado como fator a se considerar na análise, a influência da alta radiação solar na região ( $\mathrm{N} \mathrm{sol}=7,20 \pm 3,68$ horas/ sol) pelo favorecimento à degradação fotoquímica dos pesticidas. Desta forma, existe a probabilidade de redução da meia-vida destes compostos no solo em relação aos dados médios encontrados na literatura que poderá favorecer a região na dissipação dos compostos. Além disso, a alta evaporação na região $(7,64 \pm 3,25 \mathrm{~mm} /$ dia) e as temperaturas médias registradas (Tméd $=26,3 \pm 10^{\circ} \mathrm{C}$ ) poderão favorecer os processos de volatilização das moléculas.

\section{CONCLUSÃO}

Os resultados indicam que, dentre os pesticidas usados, os que apresentam maior mobilidade no ambiente são: Benomil, Dimetoato, Diuron, Fenarimol, Fention, Glifosato, Iprodione, Mancozeb, Maneb, Metalaxil, Metamidofós, Metomil, Monocrotofós, Paclobutrazol, Plocloraz, Paraquat, Tebuconazole, Tiofanato metil e Triclorfon.

As características físicas dos solos predominantes na área estudada reforçam a necessidade de pesquisas mais detalhadas da dinâmica destes produtos no perfil vertical de solos tipo areia quartzosa e vertissolos, dado o potencial de lixiviação e a profundidade dos lençóis 
subterrâneos locais (a partir de 1,5 m de profundidade).

A importância de realização de estudos para determinação do coeficiente de adsorção e da meia-vida dos produtos nos solos e temperaturas da região também foi ressaltada, assim como a necessidade de disponibilização rápida destas informações.

\section{Abstract \\ GROUND AND SURFACE WATER OF THE REGIONS PETROLINA (PE) AND JUAZEIRO (BA)}

The contamination potential of ground water and surface water in the sub-middle portion of San Francisco river basin was analyzed for pesticides applied in mango and grape cultivation by following the criteria of Environmental Protection Agency and to the index of GUS and criteria proposed by GOSS. All the criteria used take into consideration the applied products properties, by not demanding high costs nor a long time for rising information and evaluating contamination potential. The results obtained reinforce the importance of information publication on the physicochemical properties of pesticides, especially data on adsorption coefficient, whose values allow to predict the pesticide mobility in soils. This factor combined with the pesticide degradation time to the half of its initial concentration (half life) in the soil, provides information on pesticide water contamination potential. The results of this work allow the identification of the pesticides with higher contamination potential to water resources, which should be prioritized in environmental monitoring "in loco".

KEY-WORDS: GROUNDWATER; SURFACE WATER; WATER CONTAMINATION.

\section{REFERÊNCIAS}

1 COHEN, S. Z.; WAUCHOPE, R.D.; KLEIN, A.W.; EADSPORTH, C.V.; GRANCY, R. Offsite transport of pesticides in water: mathematical models of pesticide leaching and runoff. Pure and Appl. Chem., v. 67, p. 2109-2148, 1995.

2 EMBRAPA. Centro Nacional de Pesquisa em Solos. Sistema brasileiro de classificação de solos. Rio de Janeiro, 1999. $412 \mathrm{p}$.

3 EMBRAPA. Centro Nacional de Pesquisa em Meio Ambiente. Qualidade ambiental em fruticultura irrigada no nordeste brasileiro: ecofrutas. Jaguariúna, 1999a. (Projeto 11.0.99.239).

4 EMBRAPA. Centro Nacional de Pesquisa em Meio Ambiente. 
Métodos de detecção e de acompanhamento in loco dos resíduos de agrotóxicos nas frutas de manga e uva para exportação no semi-árido brasileiro: EcoFIN. Jaguariúna, 1999b. (Projeto 11.0.99.222).

FERRAZ, E.S.B. A bacia hidrográfica como unidade de estudo, estrutura e processos (apresentação). In: WORKSHOP DO PROJETO PIRACENA, 2, Piracicaba, 1996. Anais... Piracicaba: CENA, 1996. p.7.

6 FUNARI, E.; BOTTONI, P.; GIULIANO, G. Groundwater contamination by herbicides. Measured and simulated runoff volumes and peak discharges for all storms used in calibration and verification of the 1990-93 rainfall-runoff model at basin 9, Perris Valley. Processes and evaluation criteria. In: RICHARDSON, M.L. (Ed.). Chemistry agriculture and environment. Cambridge, England: The Royal Society of Chemistry, 1991. p. 235-254.

7 GUSTAFSON, D.I. Groundwater ubiquity score: a simple method for assessing pesticide leachability. Environ. Toxicol. Chem., v. 8, n. 4, p.339-357, 1989.

8 GOSS, D.W. Screening procedure for soils and pesticides for potential water quality impacts. Weed Technology, v. 6, n.3, p. 701-708, 1992.

9 MATTOS, L.M.; SILVA, E. F. da. Influência das propriedades de solos e de pesticidas no potencial de contaminação de solos e águas subterrâneas. Pesticidas: R. Ecotoxicol. e Meio Ambiente, Curitiba, v. 9, p.103-124, jan./dez. 1999.

10 SOARES, J. M. Irrigação de culturas frutíferas. Petrolina: Embrapa/CPATSA, 1995. p. 28.

11 WAUCHOPE, R.D.; BUTTLER, A.G.; HORNSBY, P.W.M.; AUGUSTIJN-BECKERS, P.W.M.; BURT, J.P. The SCS/ARS/CES pesticide properties database for environmental decision-making. Reviews of Environmental Contamination and Toxicology, v. 123, p. 1-164, 1992.

12 WORTHING, C.R.; HANCE, R.J. (Ed). The pesticide manual. $9^{\text {th }}$ ed. Great Britain: The British Crop Protection Council, 1991. p.3. 